\title{
Spread of lesions in Crohn's disease of the colon
}

F. BRAHME AND A. WENCKERT

From the Roentgen University Clinic and the Surgical University Clinic, Malmö General Hospital, University of Lund, Sweden

SUMMARY Spread of lesions in Crohn's disease of the colon was closely followed in 20 cases, representing almost one quarter of a group of surgically treated patients. Such spread occurred most commonly in an aboral direction and was usually accompanied by an exacerbation of symptoms, although spread occasionally occurred during apparently quiescent periods.

Opinions differ concerning the treatment of Crohn's disease with or without involvement of the colon (ie, regional ileitis or ileocolitis, granulomatous colitis). Conservative treatment is widely recommended (Van Patter, Bargen, Dockerty, Feldman, Mayo, and Waugh, 1954; Edwards, 1963; Lindner, Marshak, Wolf, and Janowitz, 1963; Laskin, 1964; Marshak, Lindner, and Janowitz, 1966; Crohn, 1967; Colcock and Braasch, 1968). Surgery is only considered to be indicated in cases refractory to medical treatment or when complications occur.

Factors that have contributed to this attitude are the relatively low mortality in cases treated conservatively and the high rate of postoperative recurrence. Further, the risk of spread of the lesions in Crohn's disease is generally considered insignificant, or even denied (Valdes-Dapena, 1964; Marshak et al, 1966).

Some years ago we felt that lesions of Crohn's disease spread more often than generally believed (Brahme, 1967). Experience since then has confirmed this impression and has also influenced our approach to the treatment of the disease. This paper discusses the pattern of spread of lesions in a number of cases that were followed closely, and the symptoms associated with episodes of spread.

Received for publication 16 October 1969

\section{Material}

During the last 10 years 84 patients with histologically verified Crohn's disease of the distal part of the ileum, of the distal ileum and colon, or of the colon alone, have been operated upon at the Malmö General Hospital. In 36 of these patients, who were selected for evaluation in this study, frequent radiological examinations enabled the extent of the lesions at several periods during the course of the disease to be determined. The initial radiological examinations had invariably been done within one year of the onset of symptoms.

\section{Radiological Studies}

Thirty-six patients were examined before operation on a total of 233 occasions (average 6.5 examinations/patient). In each patient the small bowel was examined one to six times and the colon from two to nine times. Six of the patients were first examined within one month of the onset of symptoms and an additional six within two months. In only nine cases was the first study not performed until more than six months after the onset of symptoms. 


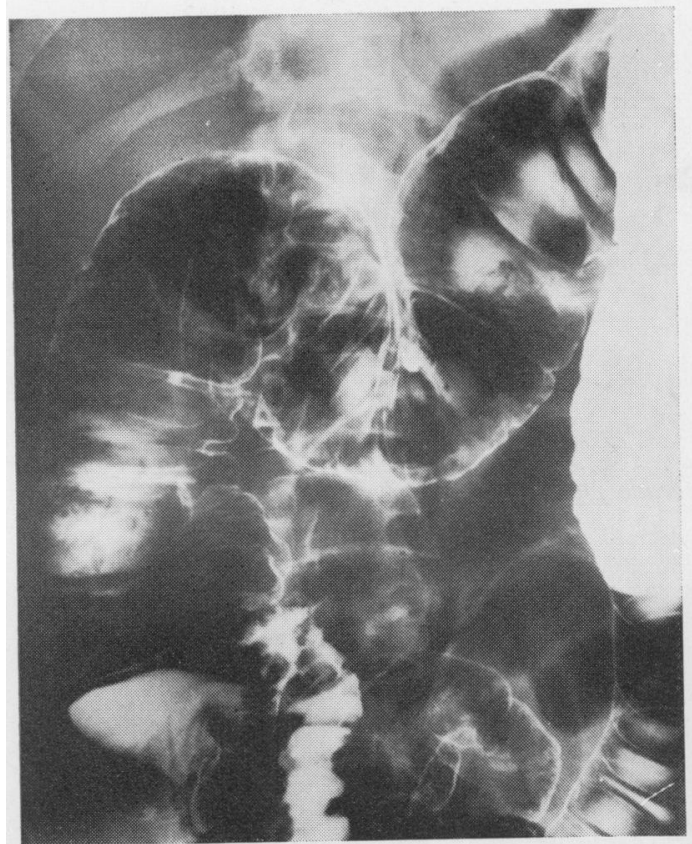

Fig. 1a.

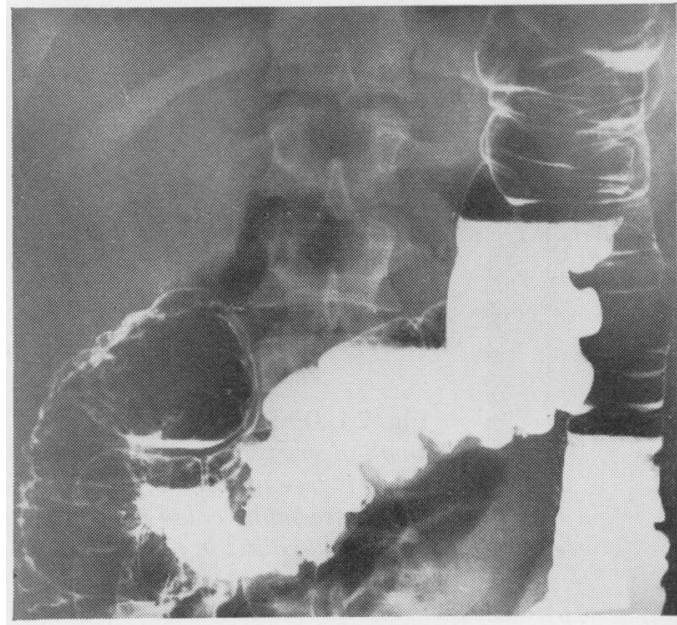

Fig. 1c.

Fig. 1 Antegrade spread. (a) Ileitis: the ascending and transverse colon is normal. (b) Two months later there are ulcers in the ascending colon. (c) Six months later, the ulcers have progressed along the transverse colon, but the splenic flexure is unaffected. (d) Nine months after the first examination: now there are also lesions in the splenic flexure, and a small group of ulcers visible in the descending colon.

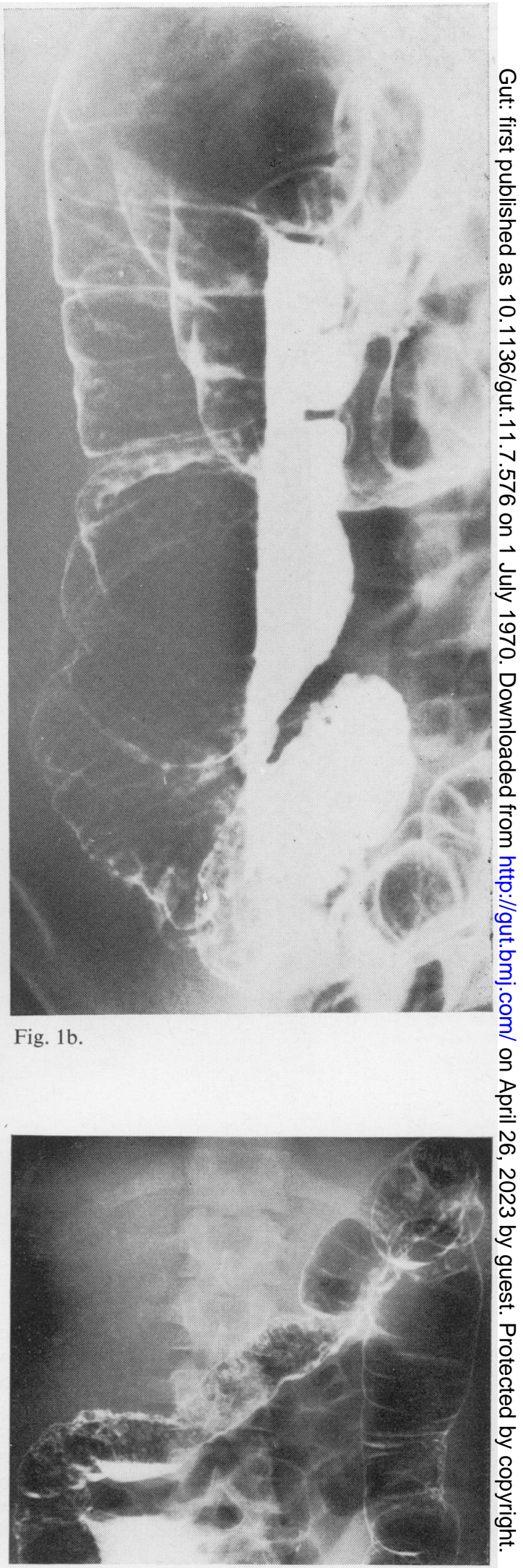

Fig. 1d. 


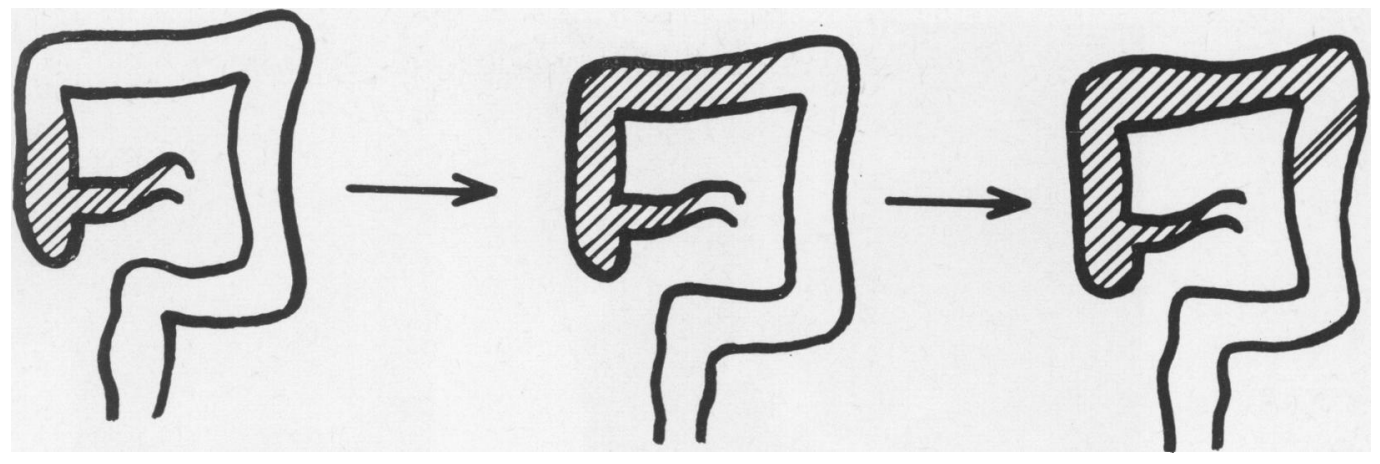

Fig. 1e. Schematic drawing of antegrade spread.

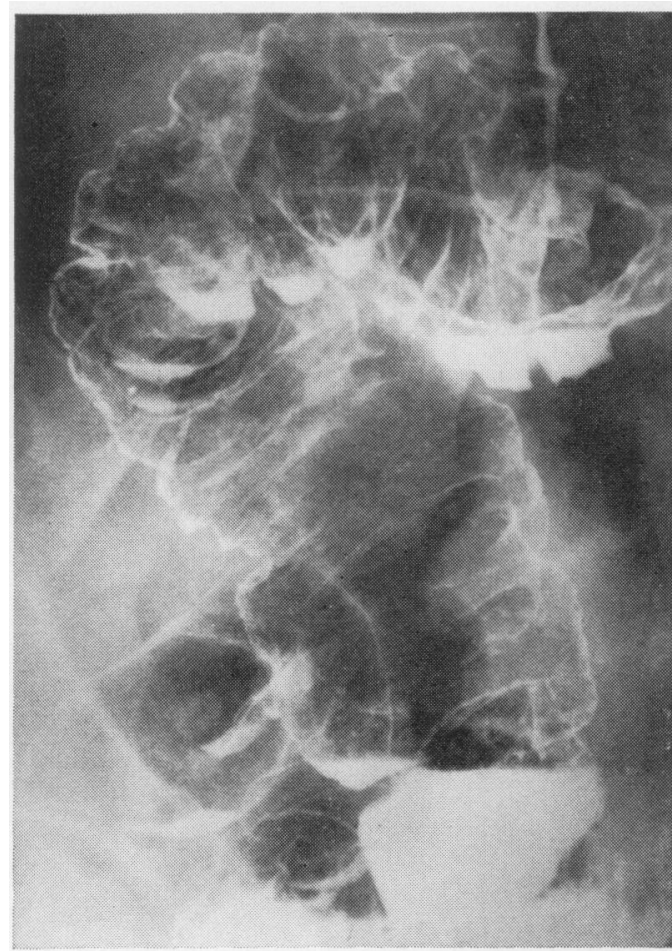

Fig. 2a.

TECHNIQUE

The small bowel was examined by the conventional follow-through technique, sometimes supplemented by retrograde examination of the small bowel. All examinations of the colon were performed by the air-contrast method, which also included taking postevacuation films. The appearance of lesions in Crohn's disease, as seen in such examinations, has been described elsewhere (Brahme, 1967) and will not be discussed in this work.

In 16 of the 36 patients, no spread of the

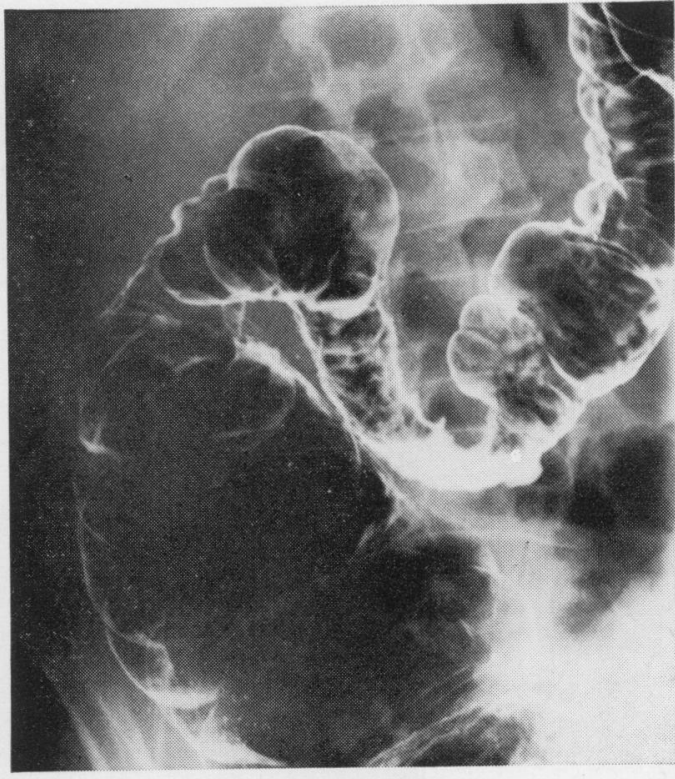

Fig. 2b.

Fig. 2 Disappearance of lesions: (a) numerous small ulcers in the ascending colon, the transverse colon is free of lesions; (b) two years later ulcers in the ascending colon have disappeared, and there is segmental colitis in the transverse colon.

lesions could be demonstrated. The lesions varied $\stackrel{\infty}{\rightarrow}$ in severity but remained confined to the same $\underline{T}$ part of the intestine during the period covered in this study. The lesions were limited to the ileo- $\stackrel{\square}{\Omega}$ caecal region in 12 patients. In the remaining $\stackrel{\mathbb{D}}{\triangle}$ four, the colon was more extensively involved. $\bar{\sigma}$ In the remaining 20 patients, spread of lesions in the colon was observed.

In 15 of these patients the lesions extended in the aboral direction. The lesions were originally $\frac{\bar{b}}{2}$ confined to the ileocaecal region in nine patients. In the six other patients larger parts of the colon 


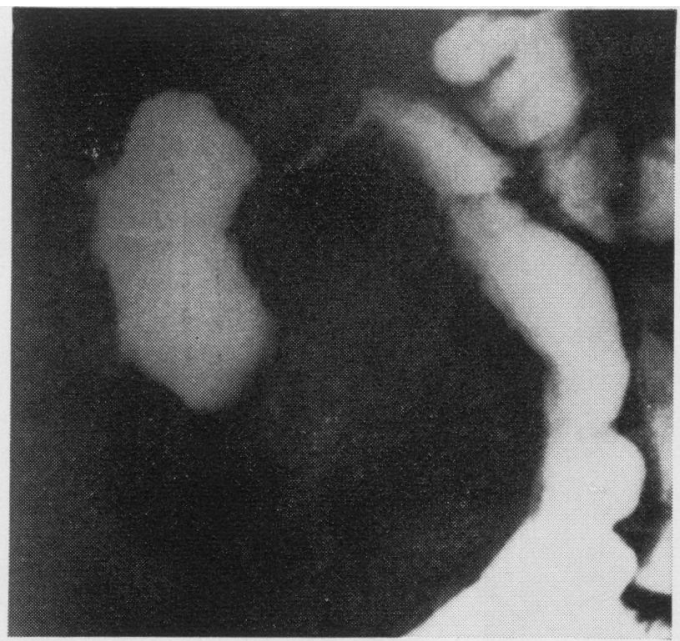

Fig. 3a.

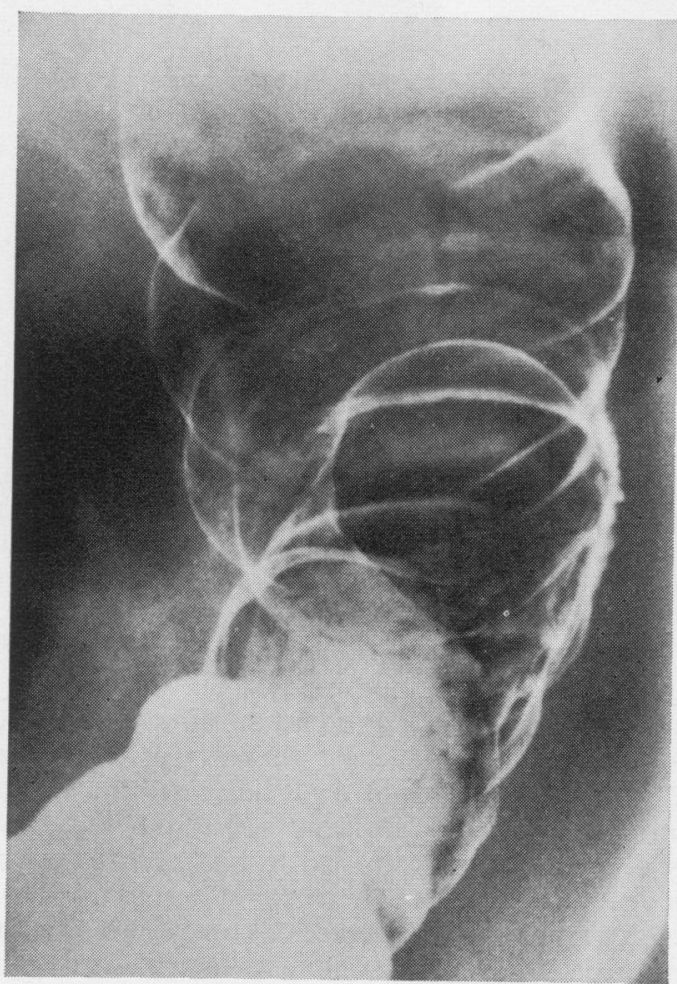

Fig. 3 Extension of 'skip' lesion: (a) ileitis and (b) small ulcers in aboral part of descending colon. The ascending and transverse colon was normal. (c) Four years later marked spread of lesions to the left. Ascending and transverse colon is still normal. N (d) Schematic drawing.

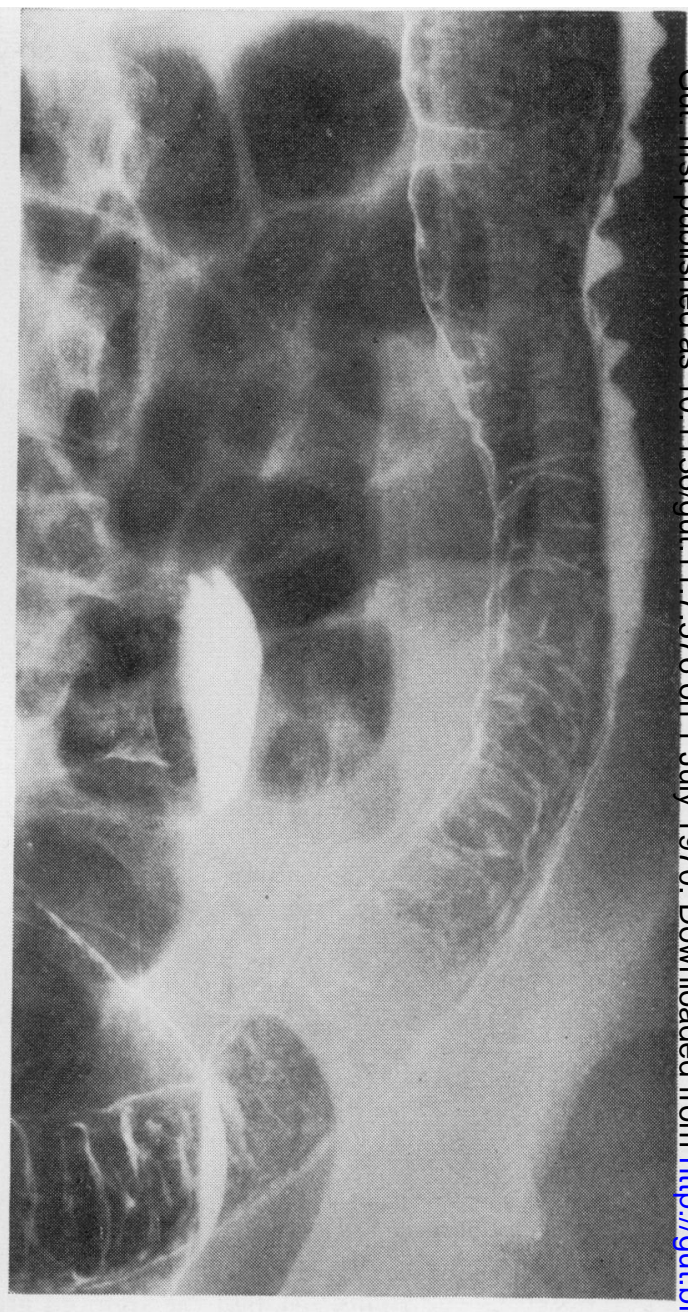

Fig. 3c.

Fig. 3b.

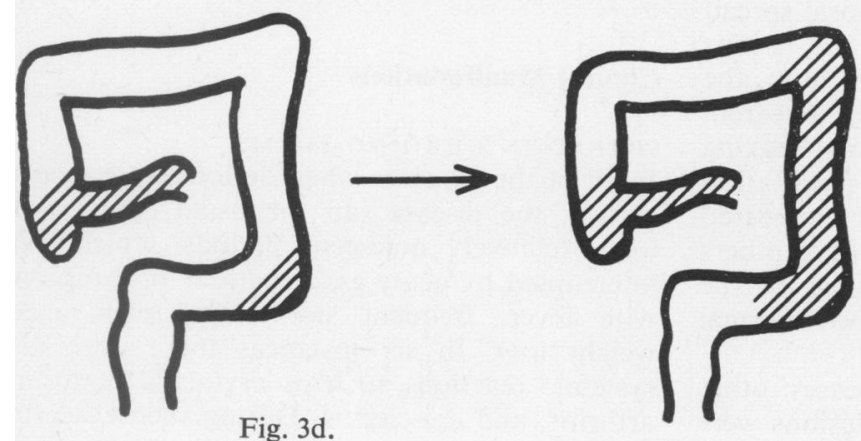

Fig. 3d. 


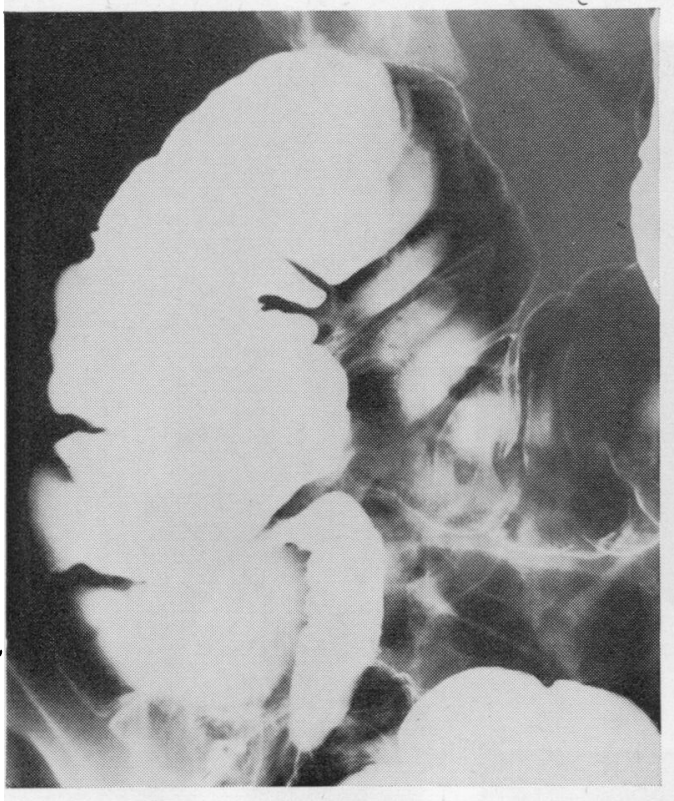

Fig. 4a.

Fig. 4 Rapid spread: (a) ileitis. The colon has a normal appearance; (b) deep ulcers in the whole colon 32 days later.

were seen to be involved at the first examination. At subsequent examinations these lesions had progressed aborally. In four patients they eventually reached the sigmoid area, and in an additional four, also the rectum. The distal end of an area where lesions had recently been present often showed tiny, barely visible ulcers, marking the limit between affected and unaffected parts of the colon (Figs. 1a-d). In none of these cases were any appreciable segments of the colon 'skipped'. Isolated groups of ulcers were sometimes found a few centimetres distal to the main lesion (Fig. 1d), but at the subsequent examination the intermediate part of the colon was usually covered with fresh lesions. This process was essentially continuous.

In five of the patients, in whom aboral spread was observed, it was noted that as the lesions extended towards the left half of the colon, the changes in the ascending caecum disappeared, leaving behind a smooth and normal-looking mucosa and apparently normal haustration (Figs. 2a and b). In some cases lesions reappeared in such areas during exacerbations, but in others radiology showed that the appearance of the segment previously affected remained normal until the time of operation.

In the remaining five of these 20 cases, other types of spread were observed. The lesions were

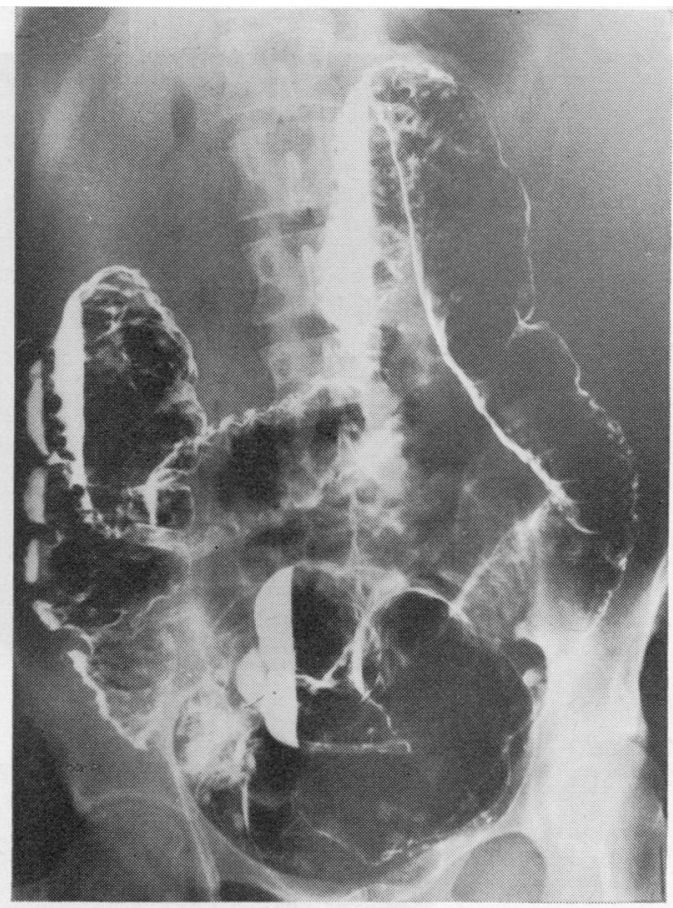

Fig. 4b.

originally found in two separate segments or 'foci', from where they later extended. In all five, the one group of lesions was situated in the distal ileum or in the ileocaecal region, the other in the descending or sigmoid colon, and that part of the colon situated between the affected areas appeared normal.

The pattern of spread of the lesions from the affected regions varied. Sometimes the lesions spread on one side, but not on the other (Figs. 3a-c) and sometimes the lesions on both sides extended in opposite directions and finally met in the transverse colon. In no instance have we observed lesions exclusively in the left half of the colon spreading in the retrograde direction.

\section{Clinical Manifestations}

\section{SPREAD IN CHRONIC FORMS}

In 17 of the 20 cases where lesions were seen to extend, the disease ran the usual course with long, relatively quiescent periods, which were interrupted by acute exacerbations of symptoms with fever, frequent stools, and pronounced weight loss. In six instances there were also systemic reactions such as erythema nodosum, arthritis, and episcleritis. During such exacerba- 
tions radiology sometimes showed only an increased severity of existing lesions but occasionally a spread of the lesions occurred in one of the forms described.

It could not be determined from the symptoms alone whether an exacerbation implied local progression or extension of lesions. Such episodes, associated with episodes of spread, occurred most commonly during the first year after the onset of symptoms, but in five patients the lesions remained stationary for more than two years before any extension occurred. Spread of the lesions was not confined to periods of exacerbation. In three patients extension was demonstrated by radiographs during clinically quiescent periods, at a time when these patients were all receiving sulphasalazine or steroid treatment. Later episodes with aggravation of the symptoms, with or without further extension of the lesions ultimately brought these patients to surgery.

\section{ACUTE FORMS}

In three patients the symptoms were severe from the onset and the clinical picture resembled that of an exacerbation in the chronic cases described above. In these cases only ileitis but no colonic changes were noted at the first examination. At the second examination of the colon performed in these three patients within six weeks of the first examination, lesions had also appeared in the colon, extending from the caecum to the rectosigmoid junction (Figs. $4 a$ and $b$ ). Anal complications occurred in all three. Operations, which were performed within two to six months,

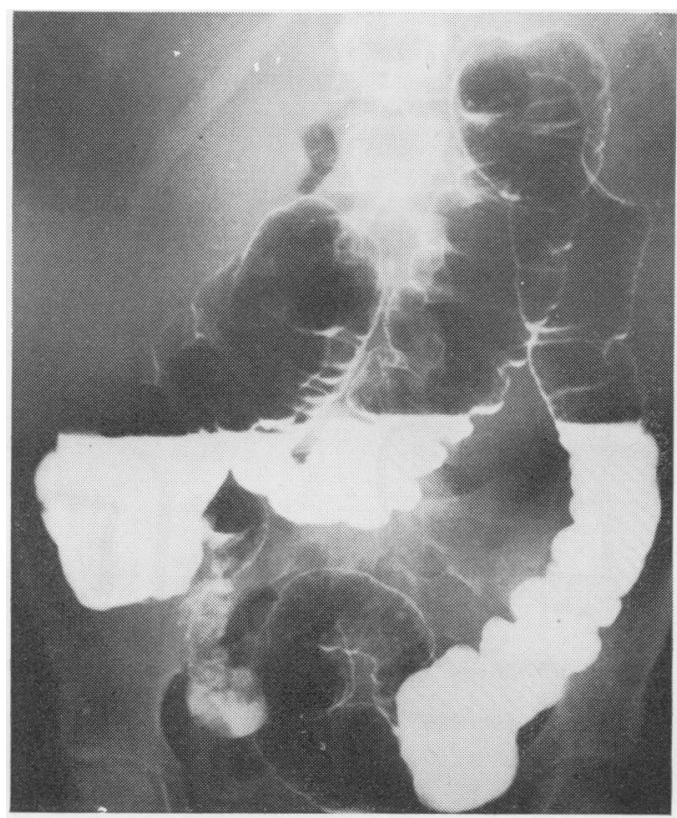

Fig. 5a. were prompted by uncontrollable diarrhoea, severe anaemia, and a generally deteriorating condition.

\section{Radiological Considerations}

A satisfactory radiological technique must beô employed to assess the extent of the lesions in $\mathbb{\widetilde { D }}$ colonic Crohn's disease. Gross lesions, as well as very small ulcers in the colon, are readily demon- ${ }^{\infty}$ strated by air-contrast technique (Brahme, 1967).. In the early stages of the disease, ulcers may not $\vec{C}$ have appeared and oedema and inflammatory infiltration of the colonic wall are then the onlyo detectable changes. Such areas may appear:normal on films of the distended colon, but, as. has been stressed by Marshak, in these cases ${ }_{i r}$ postevacuation films will demonstrate the altered pliability of the mucosa in the affected segmento (Marshak and Lindner, 1968). Consequently, films of the distended colon, as well as of the evacuated colon, are necessary to determine the $\frac{}{<}$ presence or absence of inflammatory changes in $\vec{\bullet}$ certain segments of the colon. Postevacuation $\supset$ films are routinely included in our colonic examinations and have been of help in confirm-o ing that the extension of lesions into a segment previously free of lesions definitely takes place.

In the early stages of the disease, the entire colon may be only slightly oedematous. In such cases the mucosal pattern may be difficult too을 differentiate from that seen in the normal state. Therefore, it may be that the three cases described above as 'acute forms' should be regarded as:

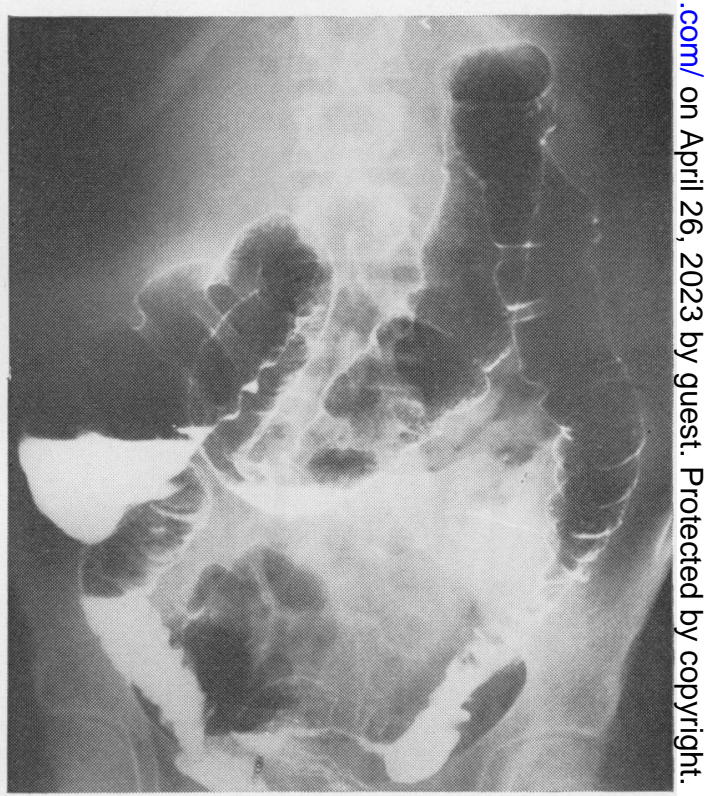

Fig. 5b. 


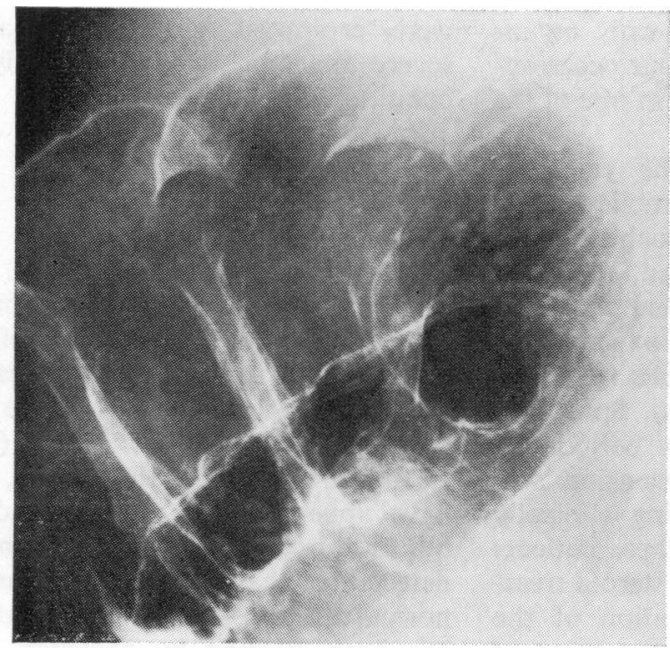

Fig. 5c.

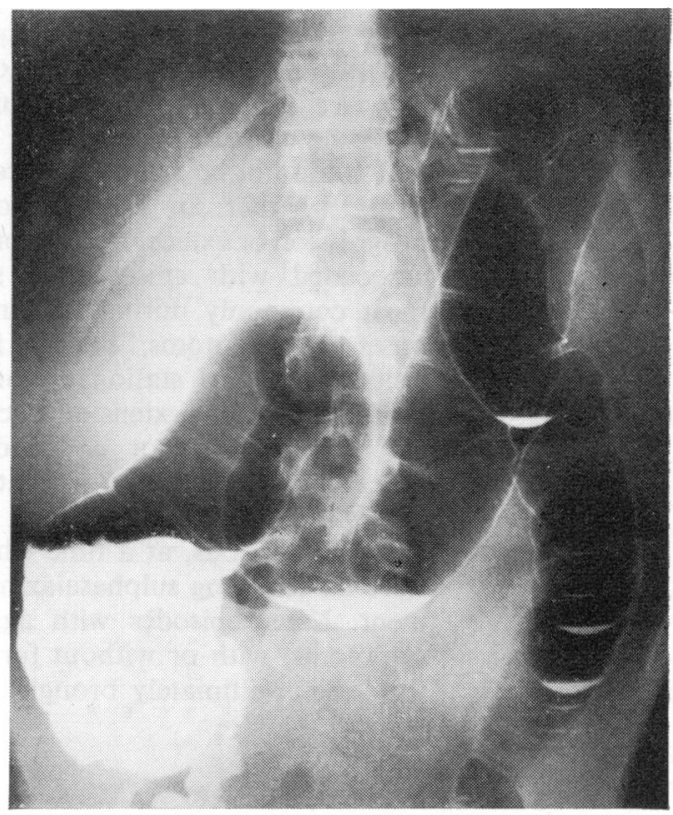

Fig. 5d.

Fig. 5 Development of rectosigmoid lesions.

(a) May 1967, ileitis, the colon has a normal appearance. (b) March 1968, extensive small ulcers in all of the colon; (c) same examination, minute ulcers in the hepatic flexure; (d) November 1968, ulcers have disappeared in ascending, transverse, and descending colon, ileitis still present; (e) January 1969 , colonic lesions confined to rectosigmoid areas. PAD: Crohn's disease with epitheloid cell granulomas; (f) schematic drawing.

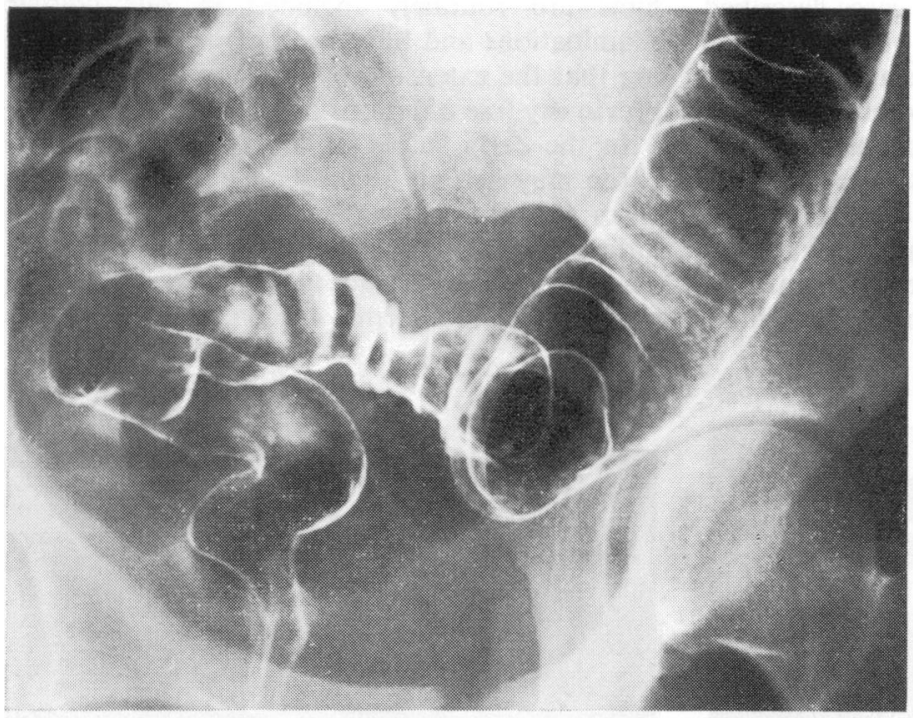

Fig. 5e.
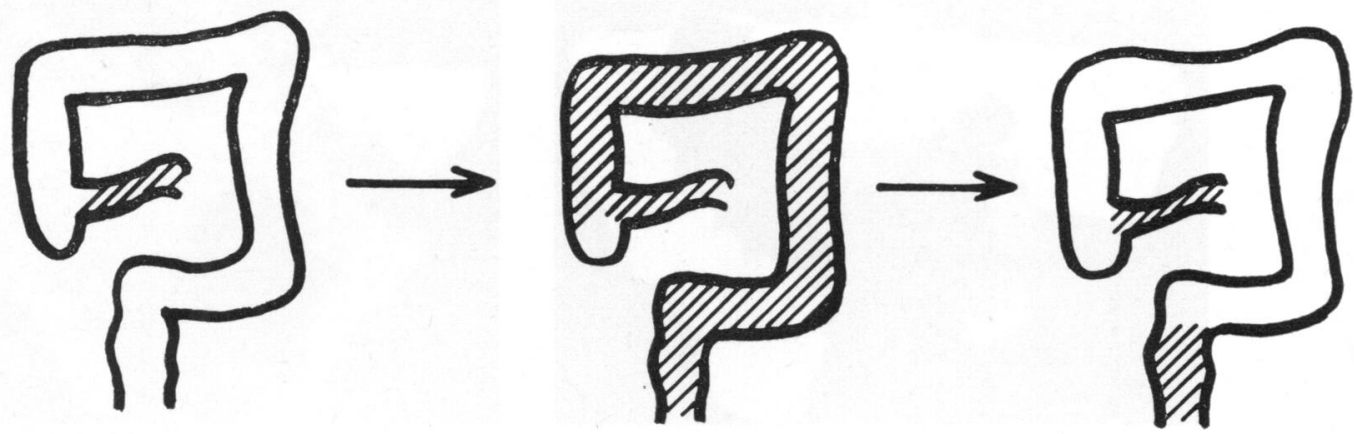

Fig. 5f. 


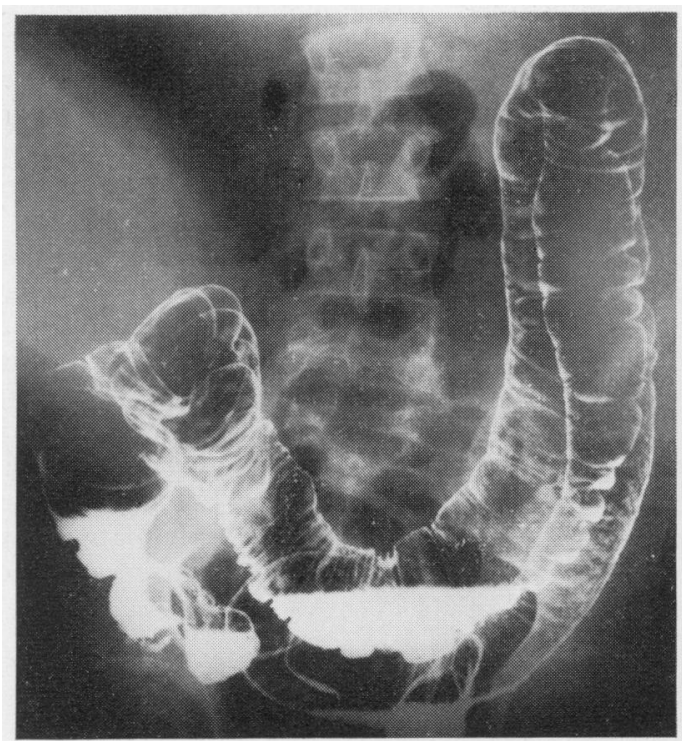

Fig. 6a.

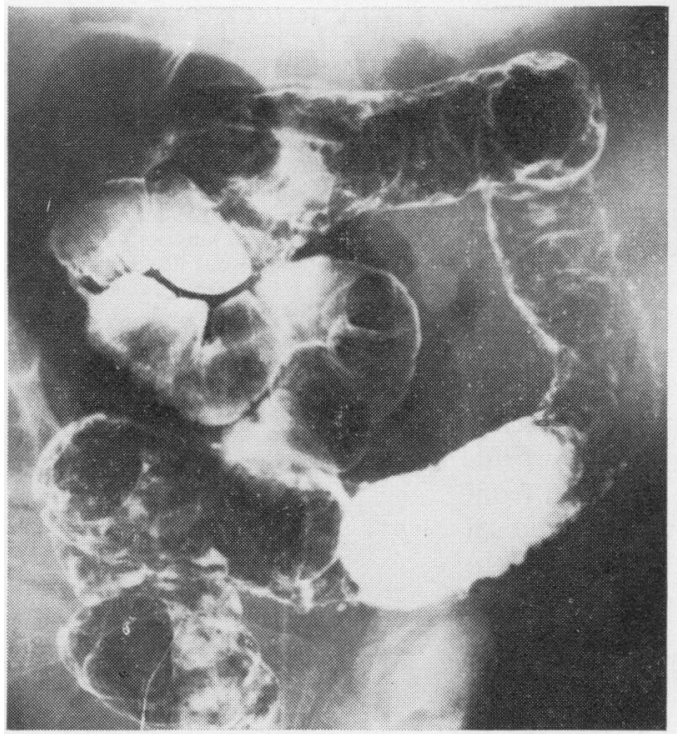

Fig. 6c.

examples of aboral spread or as cases where there is a simultaneous appearance of lesions in all segments of the colon. But in all three ileitis preceded by some weeks the changes in the colon shown by radiology. These cases have therefore been included in the group of 15 cases with 'aboral spread of lesions'.

\section{Discussion}

Spread of the lesions in Crohn's disease after

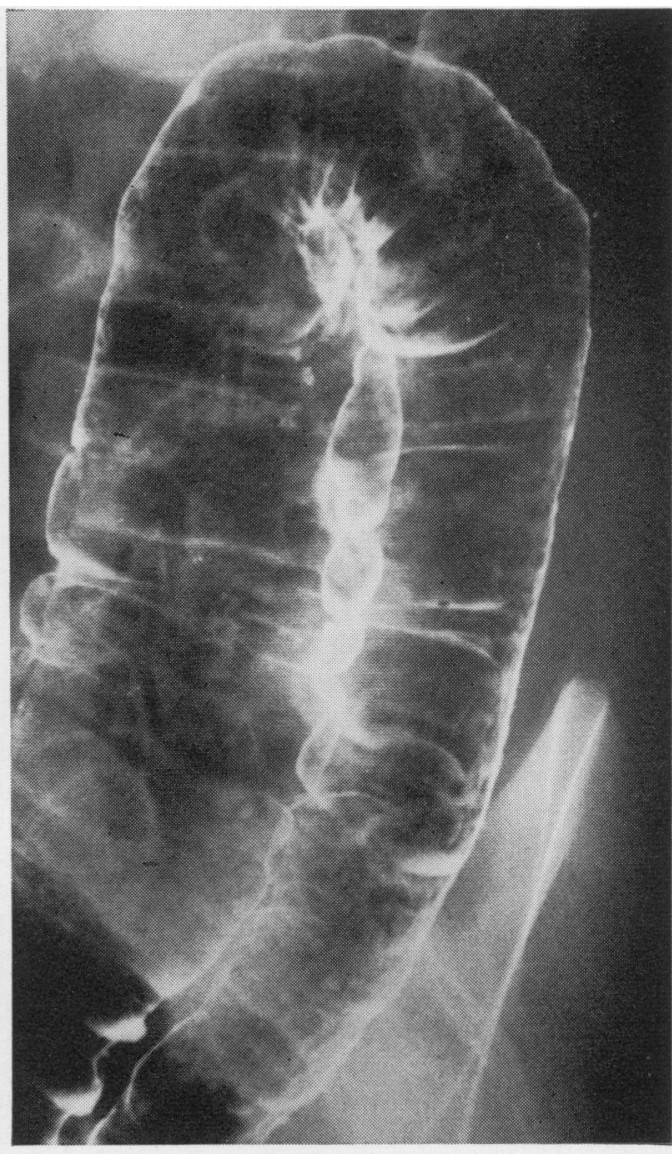

Fig. 6b.

Fig. 6 Postoperative spread: (a) marked changes in the ileocaecal region, and scattered, very small ulcers in the colon. (b) same examination: tiny lesions 3 in the splenic flexure, minute ulcers at the lateral margin of the descending colon, and small contrast flecks in shallow ulcers, (c) five months after ileocaecal resection: advanced changes in the colon and rectum.

surgical intervention has often been described $\stackrel{\oplus}{+}$ (Van Patter et al, 1954; Marshak and Lindner, 7 1968), but most authors in this field maintain that such extension occurs rarely or never in un- $\stackrel{\mathbb{Q}}{\mathbb{Q}}$ operated cases. In a series of 600 cases Marshak $\mathbb{Q}_{\mathscr{Q}}^{\mathbb{D}}$ et al (1966) noted the extension of lesions in onlyo five unoperated cases. Lockhart-Mummery and Morson (1964) did not discuss this possibilityo and Valdes-Dapena (1964) states that he had never observed the progressive creeping of the disease toward the rectum.

In our series extension of the lesions was noted 
in 20 of 84 operated patients. It was not possible to assess how often the spread of lesions actually had occurred in all the 84 patients, as many of them had not been radiologically examined until a year or more after the onset of symptoms. In other cases only one examination was done. Spread of lesions was demonstrated in 20 patients out of the selected group of 36 patients, in whom multiple radiological examinations permitted estimation of the extent of the lesions which usually occurred continuously in the aboral direction; in only five cases were other varieties of spread noted.

Of special diagnostic interest are the cases in which the disappearance of lesions in one part of the colon occurred simultaneously with the appearance of fresh lesions distally of the original site. This results in a characteristic pattern of 'segmental' colitis (Fig. 2). In one patient who originally presented with ileitis and later showed scattered, minute lesions in the proximal colon with an unaffected rectum, the lesions in the right half of the colon successively disappeared. After one and a half years of observation ileitis was still present, but colonic lesions were demonstrated only in the sigmoid and in the originally normal rectum (Figs. 5a-e). The distribution of the lesions in the colon at this stage suggested those commonly seen in ulcerative colitis; however, Crohn's disease was diagnosed histologically.

The fact that Crohn's disease of the ileum and colon may commonly migrate is not only of theoretical interest but should also be considered when conservative treatment is weighed against surgery. It is important to know that the lesions can extend not only at any period of exacerbation, the most common observation in our patients, but also when the symptoms are controlled by chemotherapy or by steroid treatment. In none of our cases could it be predicted from the initial radiological examination or the clinical findings that the lesions were later going to extend.
The possibility of a recent distal extension of lesions should be recognized before surgery is undertaken. If the patient has not been examined by radiology shortly before operation, the surgeon might not include all of the diseased bowel at resection. The minute lesions in recently involved segments of the colon do not cause hyperaemia of the serosal surface, or palpable thickening of the colon. In two of our patients, areas with small ulcers had been overlooked in the $x$-ray films and were left behind at operation (Figs. $6 \mathrm{a}-\mathrm{c})$, and the symptoms recurred within three months of the operation. At which time radiology showed substantial extension of lesions in the ileum and colon. Such incomplete resection can probably explain at least a part of the many postoperative recurrences on record.

\section{References}

Brahme, F. (1967). Granulomatous colitis. Amer. J. Roentgenol., 99, 35-44.

Colcock, B. P., and Braasch, J. W. (1968). Surgery of the Small Intestine in the Adult. Saunders, Philadelphia.

Crohn, B. B. (1967). Granulomatous diseases of the small and large bowel. Gastroenterology, 52, 767-772.

Edwards, H. (1963). Crohn's disease. J. roy. Coll. Surg. Edin., 9, $115-127$.

Laskin, M. M. (1964). Surgical management of regional enteritis. Canad. med. Ass. J., 91, 27-29.

Lindner, A. E., Marshak, R. H., Wolf, B. S., and Janowitz, H. D. (1963). Granulomatous colitis; a clinical study. New Engl. J. Med., 269, 379-385.

Lockhart-Mummery, H. E., and Morson, B. C. (1964). Crohn's disease of the large intestine. Gut, 5, 493-509.

Marshak, R. H., Lindner, A. E., and Janowitz, H. D. (1966). Granulomatous ileocolitis. Gut, 7, 258-264.

Marshak, R. H., and A. E. Lindner (1968). Granulomatous colitis. Semin. Roentgen., 3, 27-61.

Valdes-Dapena, A. (1964). Regional enteritis: pathology. In Gastroenterology, vol. II, edited by H. L. Bockhus, 2nd ed., pp. 232-247. Saunders, Philadelphia.

Patter, Van W. N., Bargen, J. A., Dockerty, M. B., Feldman, W. H., Mayo, C. W., and Waugh, J. M. (1954). Regional enteritis. Gastroenterology, 26, 347-450. 